\title{
Characterization with hybrid imaging of cystic pheochromocytomas: correlation with pathology
}

\author{
Roberta Galatola ${ }^{1}$, Valeria Romeo ${ }^{1}$, Chiara Simeoli ${ }^{2}$, Elia Guadagno ${ }^{1}$, Ilaria De Rosa ${ }^{3}$, Luca Basso ${ }^{4}$, \\ Ciro Mainolfi ${ }^{1}$, Michele Klain ${ }^{1}$, Emanuele Nicolai ${ }^{4}$, Annamaria Colao ${ }^{2}$, Simone Maurea ${ }^{1}$, Marco Salvatore ${ }^{4}$ \\ ${ }^{1}$ Department of Advanced Biomedical Sciences, University of Naples "Federico II", Naples, Italy; ${ }^{2}$ Department of Clinical Medicine and Surgery, \\ Division of Endocrinology, University of Naples "Federico II", Naples Italy; ${ }^{3}$ UOC di Anatomia ed Istologia Patologica, Azienda Ospedaliera dei \\ Colli, Naples, Italy; ${ }^{4}$ IRCCS SDN, Naples, Italy \\ Correspondence to: Valeria Romeo, MD, PhD. Department of Advanced Biomedical Sciences, University of Naples Federico II, Via S. Pansini, 5, \\ 80123, Naples, Italy. Email: valeria.romeo@unina.it.
}

Submitted Mar 26, 2020. Accepted for publication Aug 24, 2020.

doi: 10.21037/qims-20-490

View this article at: http://dx.doi.org/10.21037/qims-20-490

\section{Introduction}

Pheochromocytomas are rare neuroendocrine tumors derived from chromaffin cells arising from adrenal medulla, that may precipitate life-threatening hypertension or cardiac arrhythmias because of excessive catecholamine secretion (1). These tumors usually appear as solid hypervascular masses on imaging studies. Rarely, these lesions may undergo varying degrees of lesion degeneration such as cystic or intracellular lipid changes, or may be associated to the presence of necrosis, calcification and fibrosis, thus having unusual clinical as well as imaging appearances and thus mimicking other adrenal lesions (2). Of note, cystic lesions of the adrenal gland are uncommon, often asymptomatic and may also show malignant nature (3). Although cases of cystic pheochromocytoma have been previously reported (4), the diagnosis of these atypical pheochromocytomas is very challenging. We describe two cases of cystic pheochromocytomas with different clinical and morphologic features in which hybrid imaging using FDG PET/MRI or PET/CT reflected tumor characteristics; in particular, we highlighted the diagnostic pitfalls that can be encountered in imaging interpretation.

\section{Case 1}

A 57-year-old woman was admitted to our institute to characterize an adrenal incidentaloma discovered during an abdominal CT scan she underwent to investigate a gammaglobulinopathy. A quite-large $(5 \mathrm{~cm})$ cystic lesion was depicted at the level of right adrenal gland, showing a contextual fluid-fluid level with no evidence of fat tissue density. Clinical examination was completely silent. Laboratory assessment of both cortical and medullary adrenal function was performed; while the levels of cortical adrenal hormones were normal, measurement of plasma free and urinary metanephrine depicted increased level of plasma free $(889 \mathrm{pg} / \mathrm{mL}$, normal value: $0-180 \mathrm{pg} / \mathrm{mL})$ and urinary (1,480 $\mu \mathrm{g} / 24$ h, n.v. 0-390 $\mu \mathrm{g} / 24$ h) nor-metanephrine. To further characterize the adrenal lesion, an upper abdominal contrast-enhanced MRI was performed, confirming the cystic nature of the mass that showed homogeneous hypointensity on T1-weighted sequence with no evidence of bleeding and heterogeneous hyper-intensity on T2weighted sequence with a more clearly level due to the presence of a double component, fluid and corpuscular, without evidence of any septa. A peripheral rim of an early and persistent contrast enhancement was evidenced too. No loss of signal intensity was detected on chemical-shift images (CSI) (Figure 1A,B,C,D). No significant restricted diffusion of water molecules of the lesion was observed on diffusion weighed images (DWI) and on the corresponding apparent diffusion coefficient (ADC) map. This cystic lesion determined compression of the inferior vena cava, liver caudate lobe and homolateral diaphragmatic pillar. To furtherly characterize the lesion, suspecting malignancy for the large tumor size and heterogeneity, the patient 

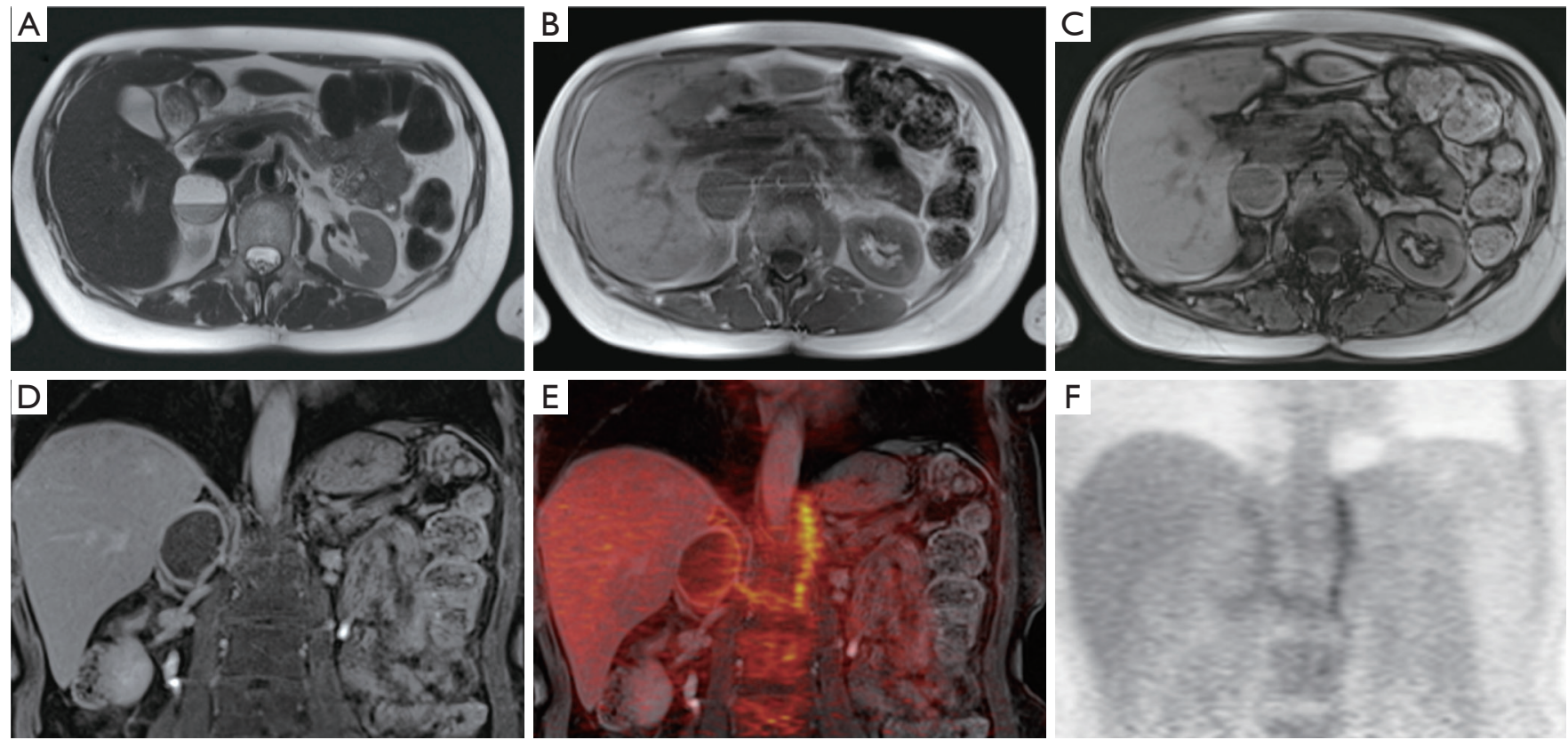

Figure 1 Contrast-enhanced ${ }^{18} \mathrm{~F}$-FDG PET/MRI axial $(\mathrm{A}, \mathrm{B}, \mathrm{C})$ and coronal $(\mathrm{D}, \mathrm{E}, \mathrm{F})$ views showing a right capsulated cystic adrenal mass with heterogeneous high signal intensity on T2-weigthed with fluid-fluid level without any evidence of septation within the mass (A); the lesion showed homogeneous low signal intensity on T1-weigthed sequence without hemorrhagic degeneration and without loss of signal intensity on CSI (B: "in-phase" image, C: "out-of-phase" image); early as well as persistent capsular enhancement was evident in postcontrast image (D: venous phase); finally, no significant tracer uptake was observed on ${ }^{18} \mathrm{~F}$ FDG PET/MRI by lesion except for faint tracer uptake along the lesion capsule (E: fusion image, F: FDG PET). CSI, chemical-shift images.

subsequently underwent a fluorine-18 (F-18) FDG-PET/ MRI that showed mainly no detectable tracer uptake by lesion except for peripheral faint tracer uptake within the capsule (Figure 1E,F). On the basis of abnormal medullary adrenal function and the presence of a large cystic adrenal mass, an atypical mainly cystic pheochromocytoma was suspected. The patient underwent right adrenalectomy with crisis of hypertension during the surgery. The pathologic sampling of the resected adrenal tumor showed a cystic pheochromocytoma. In particular, macroscopically the lesion showed no signs of angio- or fat-invasion or interruption of the capsule. Microscopically, there were a low grade of cell pleomorphism with few atypical cells $\left(<10 \mathrm{mitosis} / \mathrm{mm}^{2}\right)$; immunohistochemistry showed a low proliferative index (ki67 <2-3\%) and a positivity for S-100 protein in sustentacular cells around neuroendocrine cells, suggesting tumor benignity (Figure 2).

\section{Case 2}

A 29-year-old man was evaluated in neurologic department for clinical suspicion of multiple sclerosis. The patient had recurrent headache, visual impairment, night sweats and weight loss (10 kg in 6 mounts); moreover, arterial blood pressure was found to be slightly increased $(140 / 80 \mathrm{mmHg})$. Biochemical assessment showed abnormal medullary adrenal function with increased levels of urinary vanillylmandelic acid (12 mg/24 h, n.v. $1.8-6.7 \mathrm{mg} / 24 \mathrm{~h}$ ) and plasma free catecholamine $(250 \mathrm{pg} / \mathrm{mL}$, n.v. $0-180 \mathrm{pg} / \mathrm{mL})$. A total body contrast-enhanced CT scan was performed showing a large $(7 \mathrm{~cm})$ left inhomogeneous adrenal mass characterized by a fluid anterior portion, with spontaneous hyperdense hemorrhagic components, and a solid posterior portion. To further characterize adrenal findings, the patient underwent an abdominal contrast-enhanced MRI that confirmed the mass heterogeneity. The mostly fluid anterior component showed heterogeneous hyperintensity on T1-weighted and T2-weighted sequences suggestive of bleeding, as a complex cyst, while the solid posterior component showed high signal intensity on T2-weighted images with early and persistent contrast enhancement. No loss of signal intensity was evidenced on CSI (Figure $3 A, B, C, D$ ). 

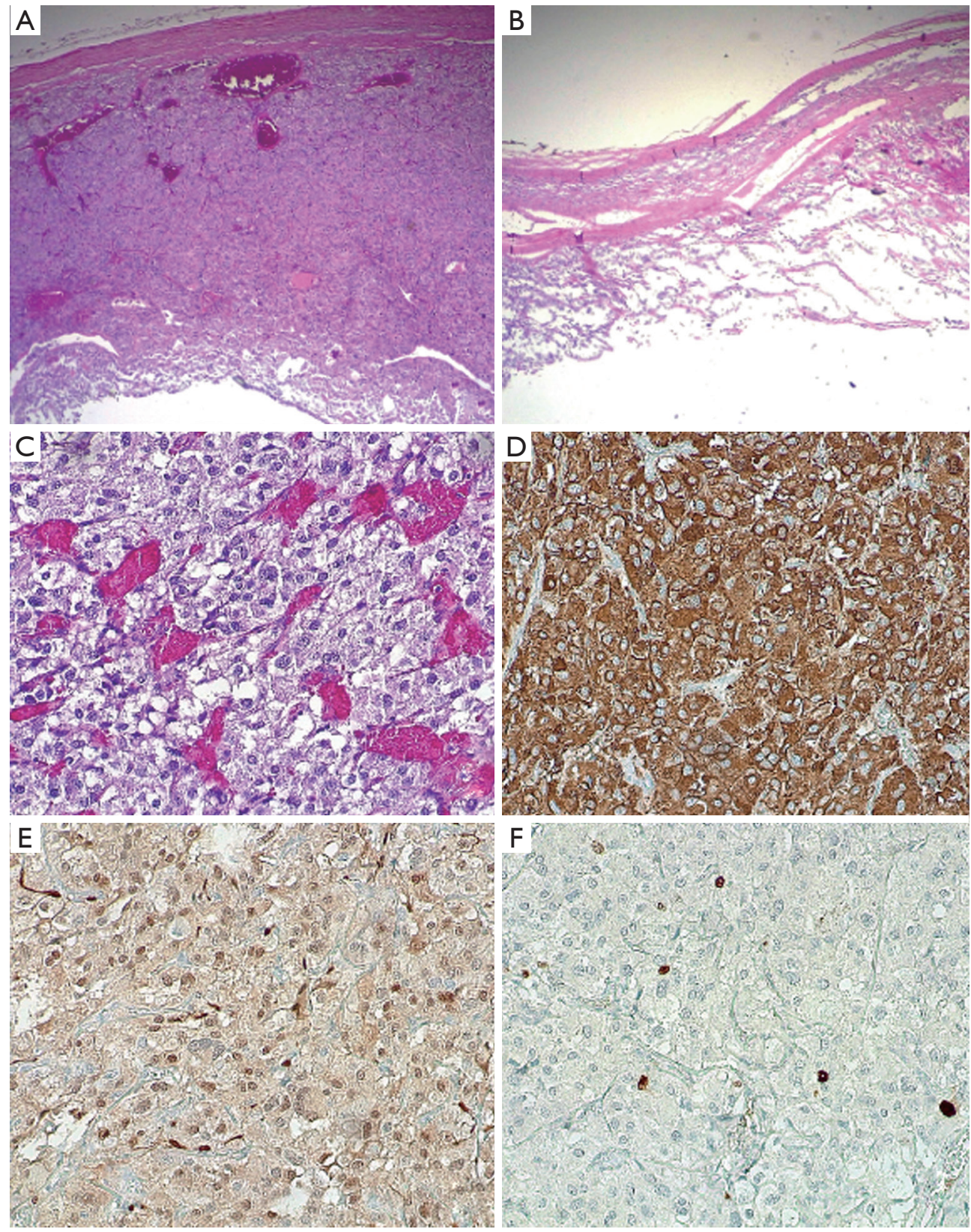

Figure 2 Microscopically, the neoplasm was mainly cystic with a variable thickness of the wall (A,B, hematoxylin and eosin stain, $25 \times$ magnification); the wall was composed by nests of medium-sized cells mixed with vascular channels and characterized by round-oval nuclei and basophilic cytoplasm (C, hematoxylin and eosin stain, 200× magnification). Immunohistochemistry showed diffuse reactivity to chromogranin in nests of neuroendocrine cells (D, 200× magnification). S100 protein was reactive in sustentacular cells around neuroendocrine cells (E, 200× magnification). Ki67 labeling index was around 2-3\% (F, 200× magnification).

Significant restricted diffusion of water molecules of the posterior portion was observed on DWI. No signs of local invasion were present. For the clinical suspicion of pheochromocytoma, iodine-123 (I-123) metaiodobenzylguanidine (MIBG) scintigraphy was performed, but no tracer uptake by the left adrenal mass was observed. Due to the suspicion of malignancy, the patient subsequently underwent F-18 FDG PET/CT that 

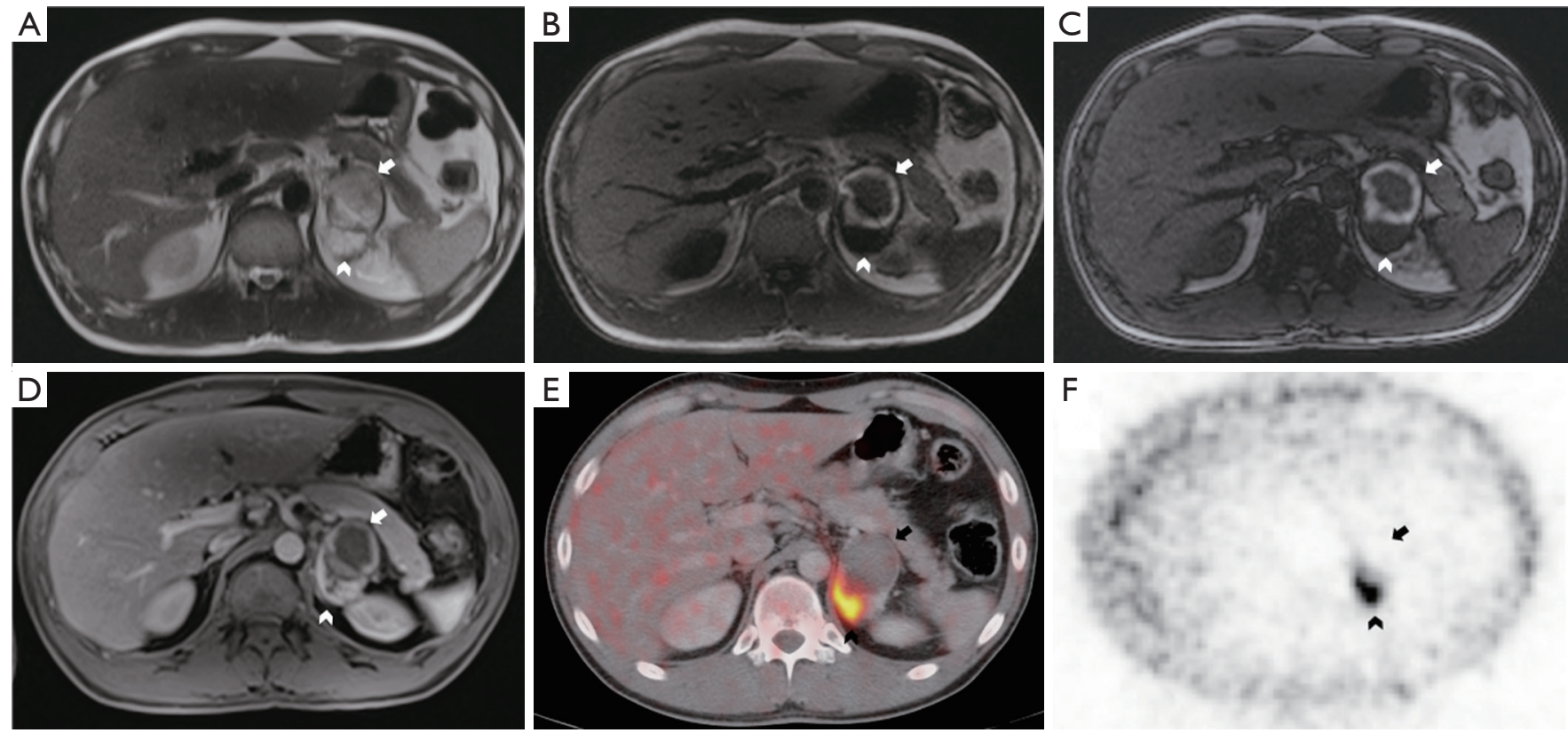

Figure 3 Contrast-enhanced axial MRI (A,B,C,D) showing a left heterogeneous adrenal mass characterized by a prevalent fluid anterior portion (arrows) with heterogeneous quite high signal intensity on T2-weighted sequence (A) and on T1-weighted sequence in periphery (B) as a complex cyst with bleeding; moreover, a solid posterior portion (arrowheads) showing high signal intensity on T2-weighted images (A) with an early and persistent contrast enhancement (D: venous phase). No loss of signal intensity was evidenced on CSI (B: "in-phase" image, C: "out-of-phase" image). Significant uptake of FDG by this solid component was detected on PET CT scan (maximum SUV: 10.5) (E: fusion image, F: FDG PET). CSI, chemical-shift images.

showed significant tracer uptake by the posterior solid portion (maximum standardized uptake value SUV: 10.5) (Figure $3 E, F$ ). On the basis of abnormal medullary adrenal function and the presence of a large mixed solid-cystic adrenal mass, an atypical partially cystic pheochromocytoma was suspected. A left adrenalectomy was performed; histopathology demonstrated a prevalent cystic pheochromocytoma with a large necrotic and hemorrhagic degeneration. However, no signs of vascular or capsule invasion were present. Immunohistochemistry showed a low proliferative index (ki67<3-4\%) and a conspicuous positivity for S-100 protein in sustentacular cells, indicative of tumor benignity (Figure 4).

\section{Discussion}

The World Health Organization (WHO) reserves the term of pheochromocytoma for catecholamine-secreting tumors arising from chromaffin cells in the adrenal medulla. This relative rare neoplasm follows "the $10 \%$ rule" being in $10 \%$ of cases in bilateral or extra-adrenal location, asymptomatic, hereditary and malignant $(2,4)$. Pheochromocytoma can precipitate cardiac arrhythmias and malignant hypertension due to adrenergic excess. Thus, if the diagnosis is overlooked, the consequences can be life threatening; however, if the diagnosis is made, affected patients are potentially curable (2). The diagnosis of pheochromocytoma is dependent on the imaging identification of an appropriately located mass together with clinical and biochemical confirmation of an excessive catecholamine secretion. Either CT or MRI is the firstline modality for characterizing and localizing suspected pheochromocytoma. On imaging studies, these tumors usually present as solid, hyper-vascular masses with high attenuation values (>10 $\mathrm{UH})$ on CT scan and "lightbulb" bright signal intensity on T2-weighted MRI $(5,6)$; moreover, their characteristic appearance on radionuclide imaging consists of unilateral focal tracer uptake using the most specific MIBG and F-18 dihydroxyphenylalanine (DOPA)-PET and/or the most sensitive F-18 FDG as radiopharmaceuticals (7-10). In particular, the role of F-18 FDG is limited in pheochromocytoma because it 

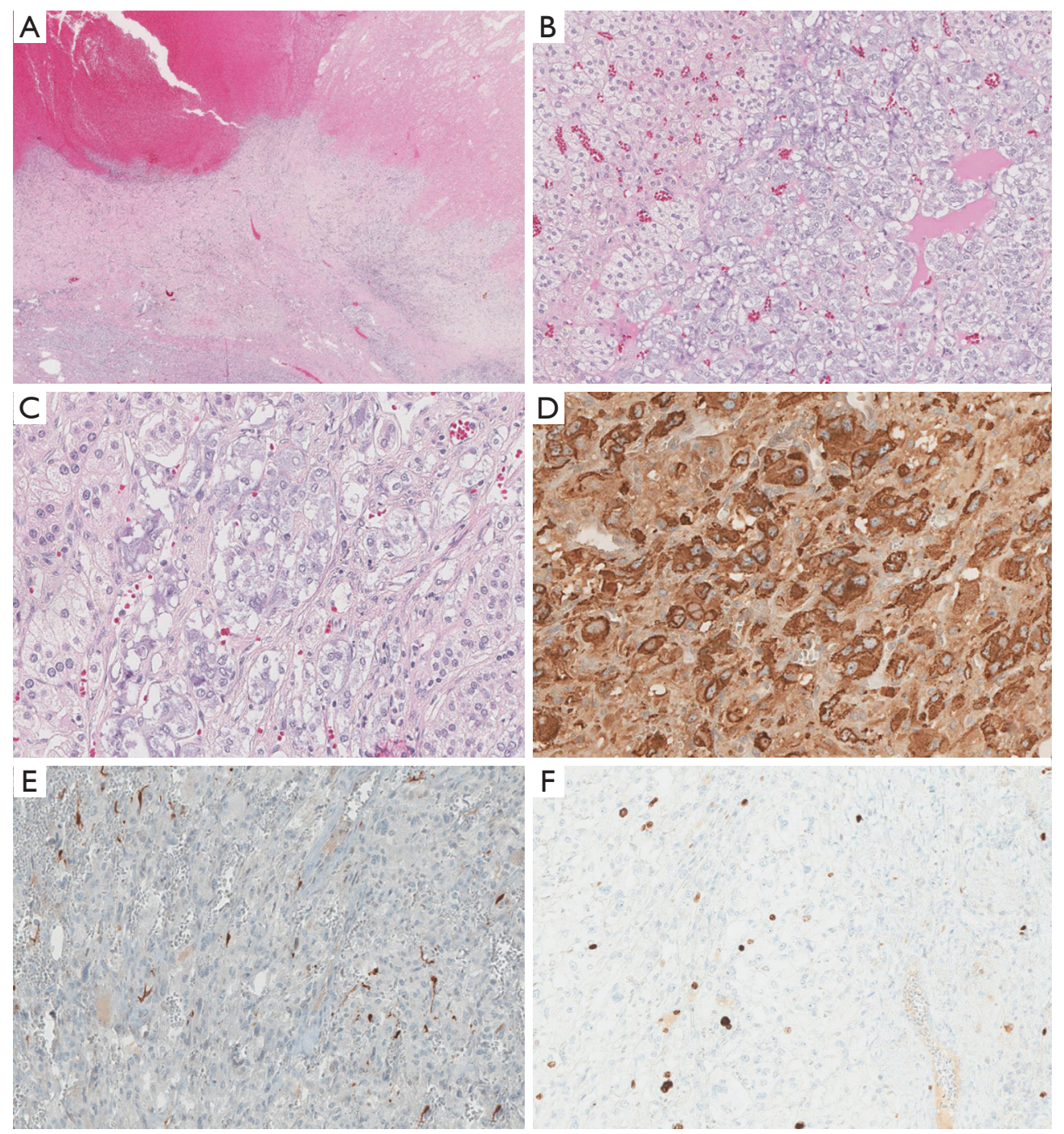

Figure 4 Microscopically, the lesion showed a large necrotic and hemorrhagic degeneration (A, hematoxylin and eosin stain, 200x magnification) as well as a solid portion composed by nests of large cells with delicate fibrovascular stroma (B, hematoxylin and eosin stain, 200x magnification) and characterized by finely granular cytoplasm with round nuclei and prominent nucleoli (C, hematoxylin and eosin stain, 80x magnification). Immunohistochemistry showed diffuse reactivity to chromogranin in nests of neuroendocrine cells (D, 70x magnification). S100 protein was reactive in sustentacular cells (E, 100× magnification). Ki67 proliferative index evaluated with MIB-1 was around $3-4 \%$ (F, 200× magnification).

does not help in tumor diagnosis which is based on the disturbances of the hormonal tests and the presence of an adrenal tumor on morphological imaging; this means that F-18 FDG is not a specific tracer for this tumor-type. The potential role of F-18 FDG in such tumor resides meanly in the evaluation of extent of the disease, such as multiple lesions i.e. paragangliomas or malignant forms; moreover, F-18 FDG is able to reflect the viable/solid component of tumor lesion when tissue degeneration is found. Finally, it has been shown that F-18 FDG may be useful in tumor lesion when the existence of a genetic mutation of cluster 1 occurs. On the other hand, DOPA-PET is highly sensitive in patients with head/neck paragangliomas or with succinate dehydrogenase subunit genes (SDH) mutations and/or biochemically silent pheochromocytomas/paragangliomas.

However, pheochromocytoma may undergo varying degrees of lesion degeneration such as necrosis, calcification, fibrosis, cystic or intracellular lipid changes, 
creating a broad spectrum of imaging appearances that may mimic other adrenal lesions, both benign and malignant, thus earning the epithet of "an imaging chameleon" (5). In particular, cystic degeneration may be so marked that only a few identifiable cells may remain in a thin rim or nodule that serve to disclose the true nature of the lesion (3-5); of note, wide hemorrhage followed by necrosis with cyst formation and subsequent resorption of the blood is the likely explanation for this tumor cystic changes; in details, the triggering mechanism could be the tumor outgrowing its vascular supply. This cystic degeneration has been reported not only in pheochromocytoma, but also in other primary adrenal neoplasms either benign cortical adenomas and hemangioma or in malignant adrenal lesions, both primary and metastatic, particularly from breast cancer $(1,3)$. The imaging differentiation between an adrenal cyst and a cystic adrenal neoplasm is beneficial for correct patient management; currently, advanced imaging modalities may be helpful for this purpose $(5,6)$. In this study, we describe two different cases of cystic appearance of sporadic pheochromocytoma in which hybrid imaging using ${ }^{18} \mathrm{~F}-\mathrm{FDG}$ PET/MRI or PET/CT reflected tumor tissue structure; in this regard, hybrid imaging, combining anatomical and functional diagnostic information, has been demonstrated to provide a more complete insight into biological tumor process $(11,12)$.

In the first case, the pheochromocytoma was almost entirely cystic without bleeding and showed similar clinicalimaging characteristics as compared to those previously described in the largest series reported in literature such as incidental finding, asymptomatic and enhancing tumor rim associated with a central cystic mass as the foremost imaging feature (4). The significant decreased number of cells producing catecholamine and the increased interstitial tissue without bioactivity might be explain the lack of symptoms and the prevalent absence of ${ }^{18} \mathrm{~F}$-FDG uptake by this large cystic tumor. In this regard, the uptake of ${ }^{18}$ F-FDG by PET/MRI, instead of its low specificity, shows a high sensitivity for tumor detection (9), being almost a constant feature of pheochromocytomas, particularly in hereditary tumors in which false negative results of other radionuclide imaging can occur (10); hence, the faint peripheral tracer uptake in the lesion capsule might be due to the poor amount of residual viable cells, as showed by histopathological samples. Simultaneously, the majority of catecholamines and other metabolic tumor products may be stored in the lesion capsule which infuses intravenously into the blood circulation when isolating the mass, as previously suggested (13). This process could explain the increased levels of catecholamines and the sudden intraoperative hypertension crisis as occurred in our case; of note, MIBG imaging was not performed in this patient since the lesions was completely cystic. Therefore, ${ }^{18} \mathrm{~F}$-FDG PET/MRI, as hybrid imaging, reflected tumor features in terms of residual solid viable tissue showing contrast enhancement and faint FDG uptake in the capsule.

The second case shows different clinical and imaging features because this cystic pheochromocytoma showed a wider solid portion as also confirmed by pathological sampling. This solid portion of the lesion showed the typical MRI high signal intensity on T2-weighted image as well as on T1-weighted sequence after contrast administration; similarly, ${ }^{18} \mathrm{~F}$-FDG uptake was detectable on PET/CT only in this solid portion of the mass reflecting viable tumor tissue. Of note, this part of the mass could be responsible of the occurrence in this patient of typical adrenergic clinical symptoms such as recurrent headache, night sweats and arterial hypertension. Conversely, the complete absence of I-123 MIBG uptake by this lesion might be explained by the variable affinity of MIBG to the amine transport system, the variable amount of cytoplasmic storage granules and the loss of the amine transport system in dedifferentiated tumors $(9,14)$; in such cases, ${ }^{18} \mathrm{~F}$-FDG is more useful as tracer since its accumulation reflects the proliferative activity of tumors, not the amine pathways, and it is more closely associated with the amount of viable cells. Thus, ${ }^{18} \mathrm{~F}-\mathrm{FDG}$ PET/ CT, as hybrid imaging, reflected tumor features in terms of residual viable tissue showing significantly increased contrast enhancement and FDG uptake only in the solid portion of the lesion.

MIBG imaging has been widely used for the diagnostic evaluation of pheochromocytoma $(7,8)$; however, possible occurrence of false negative MIBG results is reported in the literature (10). In this regard, very small pheochromocytoma measuring less than 1 centimeter, lesions with cystic degeneration, necrosis or hemorrhage, as well as poorly differentiated tumors, head-neck paragangliomas or such tumors in hereditary syndromes may be associated with false negative images with no MIBG uptake but positive FDG uptake (10). Furthermore, it should be also underlined that some false negative MIBG findings may be a consequence of interfering drugs that are often used to control arterial hypertension and tachyarrhythmia in patients with such tumors (10).

Of note, for hereditary pheochromocytomas, the Endocrine Society Clinical Practice Guidelines do 
not recommend genetic testing in all patients because it has limited incremental value in case of unilateral pheochromocytoma, no syndromic features or malignant histopathological signs as well as no positive family history, such as occurred in our patients, in view of financial costs and negative impacts on people (15); in these cases, the low probability of hereditary pheochromocytoma, despite of the young age in patient of Case 2, leads us to not testing the patients.

Therefore, the imaging characterization of atypical cystic pheochromocytoma represents a diagnostic challenge. Nevertheless, a non-invasive pre-operative diagnosis is crucial because biopsy may trigger a cardiovascular crisis and must be avoided, Furthermore, surgeons have to be prepared to face any intraoperative complications during adrenalectomy. Thus, hybrid imaging together with biochemical evaluation are recommended to characterize such adrenal tumors, underlining that a mostly cystic mass with thin enhancing rim or solid component may represent a pheochromocytoma, even in absence of usual MIBG uptake or in presence of partial FDG accumulation. In conclusion, cystic pheochromocytomas may show atypical clinical, imaging and functional features due to variable amount of viable tumor tissue, determining diagnostic pitfalls in imaging interpretation; hybrid imaging techniques such as FDG PET/MR or PET/CT are able to precisely reflect tumor tissue structure.

\section{Acknowledgments}

Funding: None.

\section{Footnote}

Conflicts of Interest: All authors have completed the ICMJE uniform disclosure form (available at http://dx.doi. org/10.21037/qims-20-490). The authors have no conflicts of interest to declare.

Ethical Statement: The study was approved by our Institutional Review Board and written informed consent was obtained.

Open Access Statement: This is an Open Access article distributed in accordance with the Creative Commons Attribution-NonCommercial-NoDerivs 4.0 International License (CC BY-NC-ND 4.0), which permits the noncommercial replication and distribution of the article with the strict proviso that no changes or edits are made and the original work is properly cited (including links to both the formal publication through the relevant DOI and the license). See: https://creativecommons.org/licenses/by-nc-nd/4.0/.

\section{References}

1. Lattin GE Jr, Sturgill ED, Tujo CA, Marko J, SanchezMaldonado KW, Craig WD, Lack EE. From the radiologic pathology archives: Adrenal tumors and tumor-like conditions in the adult: radiologic-pathologic correlation. Radiographics 2014;34:805-29.

2. Lee TH, Slywotzky CM, Lavelle MT, Garcia RA. Cystic pheochromocytoma. Radiographics 2002;22:935-40.

3. Erickson LA, Lloyd RV, Hartman R, Thompson G. Cystic adrenal neoplasms. Cancer 2004;101:1537-44.

4. Andreoni C, Krebs RK, Bruna PC, Goldman SM, Kater CE, Alves MT, Ortiz V. Cystic phaeochromocytoma is a distinctive subgroup with special clinical, imaging and histological features that might mislead the diagnosis. BJU Int 2008;101:345-50.

5. Blake MA, Kalra MK, Maher MM, Sahani DV, Sweeney AT, Mueller PR, Hahn PF, Boland GW. Pheochromocytoma: an imaging chameleon. Radiographics 2004;24 Suppl 1:S87-99.

6. Leung K, Stamm M, Raja A, Low G. Pheochromocytoma: the range of appearances on ultrasound, CT, MRI, and functional imaging. AJR Am J Roentgenol 2013;200:370-8.

7. Maurea $\mathrm{S}$, Cuocolo A, Imbriaco M, Pellegrino T, Fusari M, Cuocolo R, Liuzzi R, Salvatore $M$. Imaging characterization of benign and malignant pheochromocytoma or paraganglioma: comparison between MIBG uptake and MR signal intensity ratio. Ann Nucl Med 2012;26:670-5.

8. Maurea S, Mainenti PP, Romeo V, Mollica C, Salvatore M. Nuclear imaging to characterize adrenal tumors: Comparison with MRI. World J Radiol 2014;6:493-501.

9. Taïeb D, Sebag F, Barlier A, Tessonnier L, Palazzo FF, Morange I, Niccoli-Sire P, Fakhry N, De Micco C, Cammilleri S, Enjalbert A, Henry JF, Mundler O. 18F-FDG avidity of pheochromocytomas and paragangliomas: a new molecular imaging signature? J Nucl Med 2009;50:711-7.

10. Taïeb D, Hicks RJ, Hindié E, Guillet BA, Avram A, Ghedini P, Timmers HJ, Scott AT, Elojeimy S, Rubello D, Virgolini IJ, Fanti S, Balogova S, Pandit-Taskar N, Pacak K. European Association of Nuclear Medicine Practice Guideline/Society of Nuclear Medicine and Molecular 
Imaging Procedure Standard 2019 for radionuclide imaging of phaeochromocytoma and paraganglioma. Eur J Nucl Med Mol Imaging 2019;46:2112-37.

11. Werner MK, Schmidt H, Schwenzer NF. MR/PET: a new challenge in hybrid imaging. AJR Am J Roentgenol 2012;199:272-7.

12. Wibmer AG, Hricak H, Ulaner GA, Weber W. Trends in oncologic hybrid imaging. Eur J Hybrid Imaging 2018;2:1.

13. Li C, Chen Y, Wang W, Teng L. A case of clinically silent giant right pheochromocytoma and review of literature. Can Urol Assoc J 2012;6:E267-9.

Cite this article as: Galatola R, Romeo V, Simeoli C, Guadagno E, De Rosa I, Basso L, Mainolfi C, Klain M, Nicolai E, Colao A, Maurea S, Salvatore M. Characterization with hybrid imaging of cystic pheochromocytomas: correlation with pathology. Quant Imaging Med Surg 2021;11(2):862-869. doi: 10.21037/qims-20-490
14. Shulkin BL, Koeppe RA, Francis IR, Deeb GM, Lloyd RV, Thompson NW. Pheochromocytomas that do not accumulate metaiodobenzylguanidine: localization with PET and administration of FDG. Radiology 1993;186:711-5.

15. Lenders JW, Duh QY, Eisenhofer G, Gimenez-Roqueplo AP, Grebe SK, Murad MH, Naruse M, Pacak K, Young WF Jr, Endocrine Society. Pheochromocytoma and paraganglioma: an endocrine society clinical practice guideline. J Clin Endocrinol Metab 2014;99:1915-42. 